\title{
Comparison of osmoprotectants and antioxidant enzymes of different wild Kentucky bluegrass in Shanxi province under
} high-temperature stress

\author{
Fangshan Xia, Zhaosheng Han, Huisen Zhu, Kuanhu Dong and Lixia Du \\ College of Grassland Science, Shanxi Agricultural University, Taigu, Shanxi, China
}

\begin{abstract}
Summary
High-temperature stress was one of the biggest threats to cool-season turfgrass, hence breeding new varieties adapted to high temperature was the fundamental way to improve the turf quality. This study was designed to determine the changes in contents of malonaldehyde (MDA), soluble sugar, soluble protein and proline, and activities of superoxide dismutase (SOD) and catalase (CAT) in different accessions of wild Kentucky bluegrass (Poa pratensis), which was collected from 13 areas of Shanxi province and subjected to $0,5,10,15,20$ and 25 days of high-temperature stress, to evaluate the adaptability of different accessions of Kentucky bluegrass to high temperature stress. The results showed that the contents of soluble sugar, proline and MDA enhanced in all accessions of Kentucky bluegrass with the increase of hightemperature stress, the soluble protein contents of all accessions of Kentucky bluegrass declined under 0 to 10 days of high-temperature stress, and then increased with the increase of high-temperature stress. Inversely, the SOD and CAT activities of all Kentucky bluegrass augmented under 0 to 10 or 15 days of hightemperature stress, but they all declined with the continuous increase of high-temperature stress. The changes in osmotic and antioxidant protective system were very different in response to high-temperature stress in different accessions of Kentucky bluegrass, and the accessions of 'Qinshui' and 'Hunyuan' had the highest resistance to high-temperature stress, hence they could be used to breed new varieties that are resistant to high-temperature stress.
\end{abstract}

Keywords

antioxidase, heat stress, lipid peroxidation, osmoregulation substances, Poa pratensis

\section{Introduction}

The events of short-term high-temperature stress have occurred frequently across the globe in recent decades and are likely to increase with human-activities climate changes (Gourdji et al., 2013). However, high-temperature stress is one of the most crucial challenges for plants, which generates serious retardation in their growth and development, and even death, hence severely reduces their yields (Rezaei et al., 2015; Tutar et al., 2017; Webber et al., 2017). Therefore, extensive agricultural losses are generated worldwide by the increase in high-temperature stress with global warming,

\section{Significance of this study}

What is already known on this subject?

- High-temperature stress is the most crucial challenge for cool-season turfgrass, and osmoprotectants and antioxidant enzymes were the traditional and principal protection for plants to resist the damage of adversity.

What are the new findings?

- The influences of high-temperature stress on osmotic and antioxidant protective systems were very different in different accessions of Kentucky bluegrass, and two wild accessions exhibited the excellent resistance to high-temperature stress.

What is the expected impact on horticulture?

- These findings might play a significant role in breeding new varieties that are resistant to high-temperature stress; thereby it might be conducive to establish high quality turf under temperature stress.

and often combine with drought or other stresses (Ergo et al., 2018; Fang et al., 2018). Understanding the impact of hightemperature stress on growth and development in plants could help to reduce the loss of agricultural production.

High-temperature stress induces a series of physiological, biochemical, and molecular changes in plants affecting their growth and development (Harsh et al., 2016). A major contributor to high-temperature stress is widely considered to be the rapid production and over-accumulation of reactive oxygen species (ROS) in plants (Mittler et al., 2011). ROS are detoxified by osmoprotectants and antioxidants in plants under normal physiological conditions (Kotak et al., 2007). Proline, soluble protein and sugar play a role in cellular osmoregulation and also exhibit many protective effects; the increase in their levels exhibits enhanced tolerance to high-temperature stress (Wu et al., 2014; Zou et al., 2016). Superoxide dismutase (SOD) is usually considered as the first line of defense against oxidative stress (Gupta et al., 2013). Catalase (CAT) scavenges $\mathrm{H}_{2} \mathrm{O}_{2}$ by breaking directly it to $\mathrm{H}_{2} \mathrm{O}$, and an increase in its activity is related to augment in stress tolerance (Kraus et al., 1995). However, the defense system cannot prevent the occurrence of oxidative stress and counterbalance the over-accumulation of ROS under environmental stress (Tutar et al., 2017). Furthermore, current researches manifest that the variation of defense system in plants had great diversity among their different genotypes under high-temperature stress (Wang and Huang, 
2004; Almeselmani et al., 2006; Harsh et al., 2016). However, studies to high-temperature stress response on model or economically important plant species showed different thermotolerance and signaling responses were discovered in those with different ecotypes (Tutar et al., 2017). Therefore, studying the impact of high-temperature stress on antioxidant capacity of different genotypes of plants could avail to breeding more adaptable varieties.

Kentucky bluegrass (Poa pratensis) is not only an important forage grass for livestock, but also is the most commonly cool-season turfgrass in temperate regions. However, high-temperature stress is the frequently encountered adversity for cool-season turfgrasses during summer, leading to a serious decline in turf quality (Wang and Huang, 2004). Decline in turf quality caused by high-temperature stress is also a momentous concern in establishment and management of Kentucky bluegrass lawn (He, 2010).

Furthermore, Kentucky bluegrass often exists in a wide range of genetic variability to stress tolerance during summer (Bonos and Murphy, 1999). Therefore, understanding their genetic variability for high-temperature stress is necessary for breeding more adaptable varieties in Kentucky bluegrass. So this study was designed to explain the difference of high-temperature resistance by determining some osmoprotectants and antioxidant enzymes of Kentucky bluegrass, thereby providing references for screening out new varieties with high-temperature resistance.

\section{Materials and methods}

\section{Seed samples}

Seeds of wild Kentucky bluegrass were collected from 13 areas of Shanxi province by the Forage Seed Laboratory of Shanxi Agricultural University in 2014, and were then sealed in plastic bags and stored at $-20^{\circ} \mathrm{C}$. Detailed collection information was shown in Table 1. 'Midnight' was prepared as the control.

\section{Seedlings cultivation}

The experiment was launched in July 2015. Seeds were uniformly sowed in plastic pots $(15 \mathrm{~cm}$ in height, $12 \mathrm{~cm}$ in diam.) filling a sterilized mixture $(3: 2: 2, \mathrm{v} / \mathrm{v})$ of soil, sand and vermiculite. Seed rate was $12 \mathrm{~g} \mathrm{~m}^{-2}$. Pots were maintained in a greenhouse for 60 days and were then moved into growth chambers for 7 days. The greenhouse had an average day/ night temperature of $21 / 14^{\circ} \mathrm{C}, 75 \%$ of relative humidity, illumination intensity of 3,000 lux, and a 12-h photoperiod. The chambers were set at $25 / 20^{\circ} \mathrm{C}$ (day/night temperature), $75 \%$ of relative humidity, illumination intensity of 3,000 lux, and a 12-h photoperiod. Pots were watered 3 times per week by using weighing method.

\section{High-temperature stress treatments}

After 7 days in growth chambers, the chambers were set at $38 / 30^{\circ} \mathrm{C}$ (day/night temperature), but relative humidity and illumination intensity remained unchanged. Each accession of Kentucky bluegrass was subjected to 6 periods of high-temperature stress $(0,5,10,15,20$ and 25 days $)$ and were prepared as the samples for the experiments. Each treatment was set 4 replicates.

\section{Osmotic materials assays}

The levels of proline contents in the samples were determined according to the method of Bates et al. (1973). The soluble sugar was estimated by anthrone method (Dubois et al., 1956). Protein contents were determined by the method of Bradford (1976) using bovine serum albumin (BSA) as the standard.

\section{Enzymes extraction and assays}

Enzymes were extracted according to Kibinza etal. (2006). $2 \mathrm{~g}$ of leaf samples were ground and homogenized using a mortar and pestle in $3.5 \mathrm{~mL}$ of potassium phosphate buffer (0.1 mol L-1, pH 7.8) containing $2 \mathrm{mmol} \mathrm{L}^{-1}$ dithiothreitol, $0.1 \mathrm{mmol} \mathrm{L}^{-1}$ ethylenediaminetetraacetic acid, $1.25 \mathrm{mmol} \mathrm{L}^{-1}$ polyethyleneglycol-4000 and $20 \%$ polyvinylpolypyrrolidone. The homogenate was centrifuged at $15.000 \mathrm{~g}$ for 15 minutes. The resulting supernatant was stored for analysis of SOD and CAT activities. All steps of the extraction procedure were performed between 1 and $4^{\circ} \mathrm{C}$.

SOD was assayed by measuring its ability to inhibit the photochemical reduction of nitro blue tetrazolium (Rao and Sresty, 2000). One unit of the enzyme activity was defined as the amount of enzyme required to result in a $50 \%$ inhibition of the rate of NBT reduction at $560 \mathrm{~nm}$.

CAT activity was measured by the dynamic change in absorbance at $240 \mathrm{~nm}$ over one minute due to the decline of extinction of $\mathrm{H}_{2} \mathrm{O}_{2}$. Supernatant $(100 \mu \mathrm{L})$ was mixed with

TABLE 1. General information of different accessions of wild Kentucky bluegrass.

\begin{tabular}{|c|c|c|c|c|c|}
\hline \multirow{2}{*}{ Accessions } & \multirow{2}{*}{ Collecting location } & \multicolumn{4}{|c|}{ Geographic location } \\
\hline & & Longitude & Latitude & Elevation (m) & Habitat \\
\hline Qingshui & Dongda Village, Qinshui County & $112^{\circ} 26^{\prime} 16.9^{\prime \prime} \mathrm{E}$ & $35^{\circ} 42^{\prime} 44.3^{\prime \prime} \mathrm{N}$ & 622 & Roadside \\
\hline Huozhou & Qiliyu Village, Huozhou City & $112^{\circ} 00^{\prime} 4.9^{\prime \prime} \mathrm{E}$ & $36^{\circ} 36^{\prime} 59.6^{\prime \prime} \mathrm{N}$ & 1,549 & Hillside \\
\hline Xinzhou & NipoVillage, Xinzhou City & $112^{\circ} 26^{\prime} 01.1^{\prime \prime} \mathrm{E}$ & $38^{\circ} 37^{\prime} 58.1 " \mathrm{~N}$ & 1,547 & Fieldside \\
\hline Wutai & Xialiuyuan Village, Wutai County & $113^{\circ} 27^{\prime} 38.6^{\prime \prime} \mathrm{E}$ & $38^{\circ} 56^{\prime} 34.4^{\prime \prime} \mathrm{N}$ & 1,509 & Valley \\
\hline Lingshi & Shigaoshan Village, Lingshi County & $111^{\circ} 00^{\prime} 55.7^{\prime \prime} \mathrm{E}$ & $36^{\circ} 00^{\prime} 44.7^{\prime \prime} \mathrm{N}$ & 1,127 & Riverside \\
\hline Lingqiu & Shangchehe Village, Lingqiu County & $114^{\circ} 18^{\prime} 46.9^{\prime \prime} \mathrm{E}$ & $39^{\circ} 20^{\prime} 06.4^{\prime \prime} \mathrm{N}$ & 852 & Valley \\
\hline Guangling & Nangeya Village, Guangling County & $114^{\circ} 15^{\prime} 27.1^{\prime \prime} \mathrm{E}$ & $39^{\circ} 37^{\prime} 57.1^{\prime \prime} \mathrm{N}$ & 1,298 & Valley \\
\hline Huanyuan & Xuankongsi Village, Hunyuan County & $113^{\circ} 42^{\prime} 32.6^{\prime \prime} \mathrm{E}$ & $39^{\circ} 39^{\prime} 41.5^{\prime \prime} \mathrm{N}$ & 1,184 & Roadside \\
\hline Ningwu & Qujiagou Village, Ningwu County & $111^{\circ} 56^{\prime} 34.3 " \mathrm{E}$ & $38^{\circ} 52^{\prime} 51.2^{\prime \prime} \mathrm{N}$ & 2,272 & Roadside \\
\hline Wuzhai & Dianping Village, Wuzhai County & $111^{\circ} 54^{\prime} 27.1 ” \mathrm{E}$ & $38^{\circ} 47^{\prime} 24.1^{\prime \prime} \mathrm{N}$ & 1,865 & Riverside \\
\hline Tunliu & Goukou Village, Tunliu County & $112^{\circ} 34^{\prime} 44.2^{\prime \prime} \mathrm{E}$ & $36^{\circ} 22^{\prime} 18.1 ” \mathrm{~N}$ & 1,000 & Riverside \\
\hline Yingxian & Ximizhuang Village, Yingxian County & $113^{\circ} 07^{\prime} 32.6^{\prime \prime} \mathrm{E}$ & $39^{\circ} 36^{\prime} 33.6^{\prime \prime} \mathrm{N}$ & 993 & Riverside \\
\hline Youyu & Mayinghe Village, Youyu County & $112^{\circ} 19^{\prime} 46.0^{\prime \prime} \mathrm{E}$ & $40^{\circ} 12^{\prime} 20.1 " \mathrm{~N}$ & 1,267 & Roadside \\
\hline
\end{tabular}


3.4 $\mathrm{mL}$ phosphate buffer $\left(25 \mathrm{mmol} \mathrm{L} \mathrm{m}^{-1}, \mathrm{pH} 7.0\right.$, mixed with $0.1 \mathrm{mmol} \mathrm{L}^{-1}$ EDTA) and $200 \mu \mathrm{L} 0.1 \mathrm{~mol} \mathrm{~L}^{-1} \mathrm{H}_{2} \mathrm{O}_{2}$. The reaction was started by adding $\mathrm{H}_{2} \mathrm{O}_{2}$. Catalase activity was measured using the method of Chance and Maehly (1955).

\section{MDA contents assays}

Lipid peroxidation was determined as the amount of MDA according to Bailly et al. (1996). $1 \mathrm{~g}$ of samples were ground in $5 \mathrm{~mL}$ deionised water and homogenised with an equal volume of $0.5 \%(\mathrm{w} / \mathrm{v})$ 2-thiobarbituric acid in $20 \%$ $(\mathrm{w} / \mathrm{v})$ trichloroacetic acid. The homogenate was incubated at $100^{\circ} \mathrm{C}$ for 15 minutes, then cooled immediately and centrifuged at 4,000 rpm for 10 minutes. The supernatant was used for MDA determination. MDA levels were quantified by measuring the absorbance at 532 and $600 \mathrm{~nm}$.

\section{Statistical analyses}

Comparisons of mean differences were carried out by the variance analysis using SPSS for Windows verson 13.0. Duncan's multiple range test $(P=0.05)$ was adopted to appraise the differences in physiological indicators among different treatments.

\section{Results}

\section{Changes in soluble sugar contents of different wild Kentucky bluegrass}

The soluble sugar contents of all the Kentucky bluegrass increased significantly $(P<0.05)$ with the enhancement of high-temperature stress, but they were different in different accessions under same high-temperature stress (Table 2). At 0 days of high-temperature stress, the soluble sugar contents were significantly $(P<0.05)$ higher in 'Huozhou', 'Wutai' and 'Tunliu' than in 'Midnight', but they were significantly $(P<0.05)$ lower in other accessions than in 'Midnight'. At 5 days of high-temperature stress, there was no significant $(P>0.05)$ difference between 'Hunyuan' and 'Midnight', and the soluble sugar contents in other accessions remained unchanged comparing to 'Midnight'. At 10 days of high-temperature stress, there was no significant $(P>0.05)$ difference among 'Midnight', 'Qinshui', 'Hunyuan' and 'Ningwu', but the soluble sugar contents in other accessions still remained unchanged comparing to 'Midnight'. At 15 days of high-temperature stress, the soluble sugar contents were significantly $(P<0.05)$ higher in 'Qinshui', 'Huozhou', 'Wutai', 'Hunyuan', 'Ningwu', 'Wuzhai' and 'Tunliu' than in 'Midnight', and they were still significantly $(P<0.05)$ lower in other accessions than in 'Midnight'. At 20 days of high-temperature stress, there were no significantly $(P>0.05)$ difference between ' $\mathrm{Wu}$ zhai'and 'Midnight', and then the soluble sugar contents were remarkable $(P<0.05)$ higher in 'Lingqiu' than in 'Midnight', but they still remained unchanged in other accessions comparing to 'Midnight'. At 25 days of high-temperature stress, the soluble sugar contents in 'Huozhou', 'Wutai', 'Lingqiu', 'Hunyuan', 'Ningwu' and 'Tunliu' were significantly $(P<0.05)$ higher than in 'Midnight', and no significant $(P>0.05)$ difference was observed among 'Midnight', 'Qinshui', and 'Guangling', and they were significantly $(P<0.05)$ lower in other accessions than in 'Midnight.

\section{Changes in soluble protein contents of different wild Kentucky bluegrass}

The soluble protein contents of all the Kentucky bluegrass decreased significantly $(P<0.05)$ at the initial stage of high-temperature stress, and then they increased significantly $(P<0.05)$ with the enhancement of high-temperature stress, but they were different in different accessions under same high-temperature stress (Table 3). At 0 days of high-temperature stress, the soluble protein contents were significantly $(P<0.05)$ higher in 'Lingqiu' and 'Hunyuan' than in 'Midnight', there was no significant $(P>0.05)$ difference among 'Midnight', 'Qingshui', 'Yingxian' and 'Youyu', but they were significantly $(P<0.05)$ lower in other accessions than in 'Midnight'. At 5 days of high-temperature stress, there was no significant $(P>0.05)$ difference among 'Midnight', 'Qingshui', 'Lingshi' and 'Hunyuan', but the soluble protein contents were significantly $(P<0.05)$ lower in other accessions than in 'Midnight'. At 10 days of high-temperature stress, except there was no significant $(P>0.05)$ difference among 'Midnight', 'Guangling', and 'Hunyuan', and the soluble protein contents

TABLE 2. Effects of high-temperature stress on soluble sugar contents in leaves of different wild Kentucky bluegrass (mg $\left.\mathrm{g}^{-1} \mathrm{FW}\right)$

\begin{tabular}{llccccc}
\hline \multirow{2}{*}{ Accessions } & \multicolumn{5}{c}{ Duration of high-temperature stress (days) } \\
\cline { 2 - 7 } & \multicolumn{1}{c}{0} & 5 & 10 & 15 & 20 & 25 \\
\hline Midnight & $2.37 \pm 0.030 \mathrm{Fc}$ & $2.69 \pm 0.025 \mathrm{Ed}$ & $3.18 \pm 0.040 \mathrm{Dc}$ & $3.58 \pm 0.027 \mathrm{Cf}$ & $4.15 \pm 0.038 \mathrm{Bd}$ & $4.81 \pm 0.044 \mathrm{Ad}$ \\
Qinshui & $2.11 \pm 0.022 \mathrm{Fe}$ & $2.58 \pm 0.020 \mathrm{Ee}$ & $3.17 \pm 0.026 \mathrm{Dc}$ & $3.85 \pm 0.032 \mathrm{Ccd}$ & $4.28 \pm 0.052 \mathrm{Bc}$ & $4.86 \pm 0.036 \mathrm{Ad}$ \\
Huozhou & $2.53 \pm 0.030 \mathrm{Fb}$ & $2.78 \pm 0.026 \mathrm{Ec}$ & $3.65 \pm 0.024 \mathrm{Db}$ & $4.08 \pm 0.026 \mathrm{Cb}$ & $4.52 \pm 0.035 \mathrm{Bb}$ & $5.05 \pm 0.040 \mathrm{Ac}$ \\
Xinzhou & $2.16 \pm 0.026 \mathrm{Fde}$ & $2.45 \pm 0.018 \mathrm{Ef}$ & $2.81 \pm 0.032 \mathrm{Dd}$ & $3.38 \pm 0.033 \mathrm{Cg}$ & $3.91 \pm 0.035 \mathrm{Be}$ & $4.31 \pm 0.018 \mathrm{Af}$ \\
Wutai & $2.77 \pm 0.027 \mathrm{Fa}$ & $3.33 \pm 0.026 \mathrm{Ea}$ & $3.76 \pm 0.012 \mathrm{Da}$ & $4.21 \pm 0.038 \mathrm{Ca}$ & $4.69 \pm 0.041 \mathrm{Ba}$ & $5.31 \pm 0.037 \mathrm{Ab}$ \\
Lingshi & $1.48 \pm 0.025 \mathrm{Fk}$ & $1.88 \pm 0.023 \mathrm{Ei}$ & $2.38 \pm 0.032 \mathrm{De}$ & $3.36 \pm 0.015 \mathrm{Cg}$ & $3.61 \pm 0.037 \mathrm{Bf}$ & $4.16 \pm 0.015 \mathrm{Ag}$ \\
Lingqiu & $1.83 \pm 0.029 \mathrm{Fg}$ & $2.18 \pm 0.026 \mathrm{Eg}$ & $2.83 \pm 0.015 \mathrm{Dd}$ & $3.38 \pm 0.018 \mathrm{Cg}$ & $4.48 \pm 0.036 \mathrm{Bb}$ & $5.04 \pm 0.035 \mathrm{Ac}$ \\
Guangling & $1.72 \pm 0.026 \mathrm{Fh}$ & $2.26 \pm 0.027 \mathrm{Eg}$ & $2.72 \pm 0.018 \mathrm{Dd}$ & $3.18 \pm 0.012 \mathrm{Ch}$ & $3.92 \pm 0.024 \mathrm{Be}$ & $4.75 \pm 0.039 \mathrm{Ade}$ \\
Hunyuan & $2.20 \pm 0.023 \mathrm{Fd}$ & $2.76 \pm 0.015 \mathrm{Ecd}$ & $3.20 \pm 0.046 \mathrm{Dc}$ & $3.82 \pm 0.032 \mathrm{Cde}$ & $4.59 \pm 0.018 \mathrm{Bab}$ & $5.80 \pm 0.027 \mathrm{Aa}$ \\
Ningwu & $2.03 \pm 0.029 \mathrm{Ff}$ & $2.61 \pm 0.019 \mathrm{Ee}$ & $3.12 \pm 0.026 \mathrm{Dc}$ & $3.73 \pm 0.044 \mathrm{Ce}$ & $4.56 \pm 0.062 \mathrm{Bb}$ & $5.42 \pm 0.043 \mathrm{Ab}$ \\
Wuzhai & $1.57 \pm 0.032 \mathrm{Fj}$ & $2.21 \pm 0.037 \mathrm{Eg}$ & $2.79 \pm 0.020 \mathrm{Dd}$ & $3.72 \pm 0.021 \mathrm{Ce}$ & $4.14 \pm 0.031 \mathrm{Bd}$ & $4.64 \pm 0.046 \mathrm{Ae}$ \\
Tunliu & $2.72 \pm 0.023 \mathrm{Fa}$ & $3.12 \pm 0.015 \mathrm{~Eb}$ & $3.54 \pm 0.043 \mathrm{Db}$ & $3.94 \pm 0.043 \mathrm{Cc}$ & $4.54 \pm 0.052 \mathrm{Bb}$ & $5.07 \pm 0.032 \mathrm{Ac}$ \\
Yingxian & $1.79 \pm 0.029 \mathrm{Fgh}$ & $2.17 \pm 0.018 \mathrm{Eg}$ & $2.44 \pm 0.055 \mathrm{De}$ & $3.12 \pm 0.020 \mathrm{Ch}$ & $3.83 \pm 0.050 \mathrm{Be}$ & $4.29 \pm 0.034 \mathrm{Afg}$ \\
Youyu & $1.65 \pm 0.015 \mathrm{Fi}$ & $2.07 \pm 0.029 \mathrm{Eh}$ & $2.38 \pm 0.032 \mathrm{De}$ & $2.99 \pm 0.029 \mathrm{Ci}$ & $3.69 \pm 0.024 \mathrm{Bf}$ & $4.26 \pm 0.026 \mathrm{Afg}$ \\
\hline
\end{tabular}

Different capital letters in the same row represent significant differences between treatments at the 0.05 level, different small letters in the same column represent significant differences between accessions at the 0.05 level. 
TABLE 3. Effects of high-temperature stress on the soluble protein contents in leaves of different wild Kentucky bluegrass $\left(\mathrm{mg} \mathrm{g}^{-1} \mathrm{FW}\right)$.

\begin{tabular}{llccccc}
\hline \multirow{2}{*}{ Accessions } & \multicolumn{5}{c}{ Duration of high-temperature stress (days) } \\
\cline { 2 - 7 } Midnight & 0 & 5 & 10 & 15 & 20 & 25 \\
Qingshui & $6.15 \pm 0.010 \mathrm{Dc}$ & $4.61 \pm 0.052 \mathrm{Fab}$ & $4.83 \pm 0.007 \mathrm{Eab}$ & $8.30 \pm 0.050 \mathrm{Cc}$ & $9.03 \pm 0.012 \mathrm{Bc}$ & $10.26 \pm 0.018 \mathrm{Ad}$ \\
Huozhou & $5.18 \pm 0.043 \mathrm{Cg}$ & $3.94 \pm 0.036 \mathrm{De}$ & $3.10 \pm 0.051 \mathrm{Ef}$ & $5.96 \pm 0.036 \mathrm{Bf}$ & $7.87 \pm 0.022 \mathrm{Ah}$ & $7.75 \pm 0.014 \mathrm{Ai}$ \\
Xinzhou & $5.25 \pm 0.063 \mathrm{Dfg}$ & $3.73 \pm 0.025 \mathrm{Ef}$ & $2.87 \pm 0.030 \mathrm{Fh}$ & $5.94 \pm 0.046 \mathrm{Cf}$ & $7.24 \pm 0.038 \mathrm{Bi}$ & $8.85 \pm 0.025 \mathrm{Ag}$ \\
Wutai & $5.75 \pm 0.030 \mathrm{Dd}$ & $3.73 \pm 0.033 \mathrm{Ef}$ & $3.57 \pm 0.018 \mathrm{Fe}$ & $6.82 \pm 0.024 \mathrm{Ce}$ & $8.69 \pm 0.032 \mathrm{Be}$ & $9.20 \pm 0.036 \mathrm{Af}$ \\
Lingshi & $5.53 \pm 0.040 \mathrm{De}$ & $4.70 \pm 0.029 \mathrm{Ea}$ & $3.37 \pm 0.027 \mathrm{Ff}$ & $6.81 \pm 0.022 \mathrm{Ce}$ & $8.51 \pm 0.019 \mathrm{Bf}$ & $8.80 \pm 0.052 \mathrm{Ag}$ \\
Lingqiu & $6.95 \pm 0.033 \mathrm{Ca}$ & $3.89 \pm 0.034 \mathrm{Ee}$ & $2.84 \pm 0.021 \mathrm{Fh}$ & $6.77 \pm 0.043 \mathrm{De}$ & $8.44 \pm 0.022 \mathrm{Bf}$ & $9.29 \pm 0.027 \mathrm{Af}$ \\
Guangling & $6.22 \pm 0.046 \mathrm{Dbc}$ & $4.16 \pm 0.022 \mathrm{Fd}$ & $4.77 \pm 0.035 \mathrm{~Eb}$ & $7.10 \pm 0.028 \mathrm{Cd}$ & $8.41 \pm 0.036 \mathrm{Bf}$ & $9.55 \pm 0.020 \mathrm{Ae}$ \\
Hunyuan & $6.31 \pm 0.019 \mathrm{Db}$ & $4.60 \pm 0.022 \mathrm{Eab}$ & $4.89 \pm 0.017 \mathrm{Ea}$ & $7.10 \pm 0.043 \mathrm{Cd}$ & $9.35 \pm 0.030 \mathrm{Bb}$ & $12.02 \pm 0.017 \mathrm{Aa}$ \\
Ningwu & $4.49 \pm 0.032 \mathrm{Ch}$ & $3.60 \pm 0.020 \mathrm{Dg}$ & $3.61 \pm 0.028 \mathrm{De}$ & $6.82 \pm 0.023 \mathrm{Be}$ & $7.20 \pm 0.020 \mathrm{Bi}$ & $8.37 \pm 0.015 \mathrm{Ah}$ \\
Wuzhai & $5.76 \pm 0.057 \mathrm{Dd}$ & $3.93 \pm 0.024 \mathrm{Ee}$ & $4.12 \pm 0.020 \mathrm{Ec}$ & $6.94 \pm 0.020 \mathrm{Ce}$ & $8.72 \pm 0.043 \mathrm{Be}$ & $10.51 \pm 0.036 \mathrm{Ac}$ \\
Tunliu & $5.36 \pm 0.015 \mathrm{Df}$ & $4.35 \pm 0.028 \mathrm{Ec}$ & $3.82 \pm 0.022 \mathrm{Fd}$ & $6.36 \pm 0.055 \mathrm{Cf}$ & $8.88 \pm 0.052 \mathrm{Bd}$ & $9.21 \pm 0.051 \mathrm{Af}$ \\
Yingxian & $6.24 \pm 0.008 \mathrm{Cbc}$ & $4.36 \pm 0.018 \mathrm{Dc}$ & $2.91 \pm 0.017 \mathrm{Eh}$ & $10.23 \pm 0.049 \mathrm{Ba}$ & $10.69 \pm 0.020 \mathrm{Aa}$ & $10.56 \pm 0.049 \mathrm{Ac}$ \\
Youyu & $6.18 \pm 0.041 \mathrm{Dbc}$ & $4.33 \pm 0.015 \mathrm{Ec}$ & $2.91 \pm 0.029 \mathrm{Fh}$ & $7.23 \pm 0.027 \mathrm{Cd}$ & $8.10 \pm 0.019 \mathrm{Bg}$ & $8.88 \pm 0.052 \mathrm{Ag}$ \\
\hline
\end{tabular}

Different capital letters in the same row represent significant differences between treatments at the 0.05 level, different small letters in the same column represent significant differences between accessions at the 0.05 level.

were also significantly $(P<0.05)$ lower in other accessions than in 'Midnight'. At 15 days of high-temperature stress, the soluble protein contents were significantly $(P<0.05)$ higher in 'Qingshui' and 'Yingxian' than in 'Midnight', and they were significantly $(P<0.05)$ lower in other accessions than in 'Midnight'. At 20 days of high-temperature stress, except the soluble protein contents were significantly $(P<0.05)$ higher in 'Hunyuan' and 'Yingxian' than in 'Midnight', and they were also significantly $(P<0.05)$ lower in other accessions than in 'Midnight'. At 25 days of high-temperature stress, the soluble protein contents were significantly $(P<0.05)$ higher in 'Qinshui', 'Hunyuan' and 'Yingxian' than in 'Midnight', and they were also significantly $(P<0.05)$ lower in other accessions than in 'Midnight'.

\section{Changes in proline contents of different wild Kentucky bluegrass}

The proline contents of all the Kentucky bluegrass increased significantly $(P<0.05)$ with the enhancement of high-temperature stress, but they were also different in different accessions under same high-temperature stress (Table 4). At 0 days of high-temperature stress, the proline contents were significantly $(P<0.05)$ higher in 'Qinshui', 'Huozhou', 'Lingshi' and 'Hunyuan' than in 'Midnight', and there was no significant $(P>0.05)$ difference between 'Midnight' and 'Lingqiu', but they were significantly $(P<0.05)$ lower in other accessions than in 'Midnight'. At 5 days of high-temperature stress, the proline contents were significantly $(P<0.05)$ higher in 'Qinshui', 'Guangling', 'Hunyuan' and 'Tunliu' than in 'Midnight', and there was no significant

TABLE 4. Effects of high-temperature stress on the free proline contents in leaves of different wild Kentucky bluegrass $\left(\mu \mathrm{g} \mathrm{g}^{-1} \mathrm{FW}\right)$.

\begin{tabular}{lcccccc}
\hline \multirow{2}{*}{ Accessions } & \multicolumn{5}{c}{ Duration of high-temperature stress (days) } \\
\cline { 2 - 7 } Midnight & $144.80 \pm 2.79 \mathrm{Fc}$ & $248.98 \pm 1.00 \mathrm{Ed}$ & $352.13 \pm 2.79 \mathrm{Dh}$ & $567.16 \pm 3.68 \mathrm{Cf}$ & $955.43 \pm 3.09 \mathrm{Be}$ & $1,384.20 \pm 4.69 \mathrm{Ac}$ \\
Qinshui & $155.12 \pm 2.42 \mathrm{Fb}$ & $255.86 \pm 0.66 \mathrm{Ebc}$ & $427.88 \pm 2.89 \mathrm{Def}$ & $617.28 \pm 4.58 \mathrm{Ce}$ & $791.34 \pm 0.20 \mathrm{Bi}$ & $1,357.96 \pm 4.50 \mathrm{Ad}$ \\
Huozhou & $166.88 \pm 2.21 \mathrm{Ea}$ & $112.44 \pm 1.56 \mathrm{Fj}$ & $347.72 \pm 4.20 \mathrm{Dh}$ & $534.73 \pm 2.25 \mathrm{Cf}$ & $1,123.07 \pm 3.46 \mathrm{Bb}$ & $1,344.78 \pm 4.04 \mathrm{Ad}$ \\
Xinzhou & $133.65 \pm 3.32 \mathrm{Fd}$ & $241.23 \pm 1.15 \mathrm{Ee}$ & $432.96 \pm 3.37 \mathrm{Dde}$ & $537.85 \pm 2.84 \mathrm{Cf}$ & $898.73 \pm 3.94 \mathrm{Bg}$ & $1,078.11 \pm 3.00 \mathrm{Ag}$ \\
Wutai & $127.50 \pm 1.45 \mathrm{Fde}$ & $251.25 \pm 1.79 \mathrm{Ecd}$ & $522.30 \pm 3.65 \mathrm{Db}$ & $612.64 \pm 2.88 \mathrm{Ce}$ & $827.14 \pm 3.88 \mathrm{Bh}$ & $1,287.09 \pm 3.89 \mathrm{Ae}$ \\
Lingshi & $173.57 \pm 2.06 \mathrm{Fa}$ & $222.54 \pm 1.07 \mathrm{Eg}$ & $433.36 \pm 3.82 \mathrm{Dde}$ & $779.49 \pm 3.91 \mathrm{Ca}$ & $1,058.97 \pm 4.54 \mathrm{Ac}$ & $832.92 \pm 2.61 \mathrm{Bi}$ \\
Lingqiu & $143.16 \pm 2.50 \mathrm{Fc}$ & $212.87 \pm 0.97 \mathrm{Eh}$ & $321.90 \pm 2.38 \mathrm{Di}$ & $718.60 \pm 4.95 \mathrm{Cc}$ & $1,037.30 \pm 3.98 \mathrm{Bd}$ & $1,091.47 \pm 2.27 \mathrm{Ag}$ \\
Guangling & $84.68 \pm 2.27 \mathrm{Fg}$ & $267.69 \pm 1.25 \mathrm{Ea}$ & $323.38 \pm 3.42 \mathrm{Di}$ & $534.30 \pm 3.06 \mathrm{Cf}$ & $754.63 \pm 1.50 \mathrm{Bj}$ & $979.04 \pm 1.81 \mathrm{Ah}$ \\
Hunyuan & $172.30 \pm 1.88 \mathrm{Fa}$ & $259.35 \pm 0.76 \mathrm{~Eb}$ & $458.61 \pm 3.86 \mathrm{Dc}$ & $636.41 \pm 4.89 \mathrm{Cd}$ & $830.75 \pm 2.86 \mathrm{Bh}$ & $1,082.57 \pm 3.80 \mathrm{Ag}$ \\
Ningwu & $119.90 \pm 1.05 \mathrm{Fe}$ & $214.87 \pm 1.73 \mathrm{Eh}$ & $444.06 \pm 3.11 \mathrm{Dcd}$ & $746.88 \pm 3.89 \mathrm{Cb}$ & $961.11 \pm 4.46 \mathrm{Be}$ & $1,442.53 \pm 4.18 \mathrm{Ab}$ \\
Wuzhai & $95.57 \pm 0.79 \mathrm{Ff}$ & $206.40 \pm 1.79 \mathrm{Ei}$ & $414.50 \pm 1.75 \mathrm{Df}$ & $742.23 \pm 3.32 \mathrm{Cb}$ & $1,247.85 \pm 4.96 \mathrm{Ba}$ & $1,555.60 \pm 4.72 \mathrm{Aa}$ \\
Tunliu & $118.23 \pm 2.85 \mathrm{Fe}$ & $261.29 \pm 1.32 \mathrm{~Eb}$ & $386.35 \pm 4.76 \mathrm{Dg}$ & $487.48 \pm 0.20 \mathrm{Ch}$ & $760.42 \pm 4.52 \mathrm{Bj}$ & $969.55 \pm 4.43 \mathrm{Ah}$ \\
Yingxian & $120.59 \pm 1.62 \mathrm{Fe}$ & $239.81 \pm 1.77 \mathrm{Eef}$ & $350.95 \pm 4.32 \mathrm{Dh}$ & $543.57 \pm 1.88 \mathrm{Cg}$ & $938.15 \pm 3.84 \mathrm{Bf}$ & $1,206.02 \pm 1.24 \mathrm{Af}$ \\
Youyu & $101.09 \pm 3.31 \mathrm{Ff}$ & $234.84 \pm 0.70 \mathrm{Ef}$ & $561.85 \pm 5.96 \mathrm{Da}$ & $723.94 \pm 1.68 \mathrm{Cc}$ & $1,071.87 \pm 4.96 \mathrm{Bc}$ & $1,349.38 \pm 4.04 \mathrm{Ad}$ \\
\hline
\end{tabular}

Different capital letters in the same row represent significant differences between treatments at the 0.05 level, different small letters in the same column represent significant differences between accessions at the 0.05 level. 


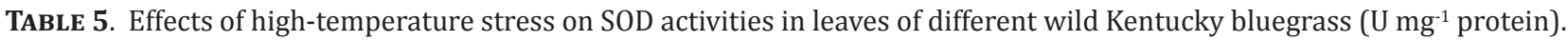

\begin{tabular}{lcccccc}
\hline \multirow{2}{*}{ Accessions } & \multicolumn{5}{c}{ Duration of high-temperature stress (days) } \\
\cline { 2 - 7 } Midnight & 0 & 5 & 10 & 15 & 20 & 25 \\
Qinshui & $109.46 \pm 0.69 \mathrm{Df}$ & $129.91 \pm 0.78 \mathrm{Bc}$ & $139.67 \pm 0.78 \mathrm{Ai}$ & $143.00 \pm 0.933 \mathrm{Af}$ & $107.51 \pm 0.90 \mathrm{Ccd}$ & $90.18 \pm 0.86 \mathrm{Dc}$ \\
Huozhou & $85.88 \pm 0.55 \mathrm{Eg}$ & $120.66 \pm 0.76 \mathrm{Cd}$ & $157.86 \pm 0.56 \mathrm{Bef}$ & $166.82 \pm 0.63 \mathrm{Ab}$ & $94.70 \pm 0.59 \mathrm{Df}$ & $42.36 \pm 0.79 \mathrm{Fj}$ \\
Xinzhou & $108.41 \pm 0.68 \mathrm{Dd}$ & $114.37 \pm 0.68 \mathrm{Ce}$ & $202.64 \pm 0.89 \mathrm{Aa}$ & $130.08 \pm 0.86 \mathrm{Bh}$ & $110.07 \pm 0.93 \mathrm{Dbc}$ & $53.20 \pm 0.56 \mathrm{Eh}$ \\
Wutai & $63.77 \pm 0.63 \mathrm{Ei}$ & $108.26 \pm 0.88 \mathrm{Cf}$ & $152.91 \pm 0.83 \mathrm{Ah}$ & $115.31 \pm 0.78 \mathrm{Bi}$ & $83.72 \pm 0.81 \mathrm{Dh}$ & $62.70 \pm 0.62 \mathrm{Eg}$ \\
Lingshi & $73.34 \pm 0.66 \mathrm{Eh}$ & $113.99 \pm 0.79 \mathrm{Ce}$ & $131.76 \pm 0.55 \mathrm{Bj}$ & $148.90 \pm 0.66 \mathrm{Ad}$ & $88.58 \pm 0.46 \mathrm{Dg}$ & $66.54 \pm 0.63 \mathrm{Ff}$ \\
Lingqiu & $97.73 \pm 0.55 \mathrm{Ee}$ & $131.75 \pm 0.83 \mathrm{Cc}$ & $154.44 \pm 0.38 \mathrm{Bgh}$ & $173.72 \pm 0.95 \mathrm{Aa}$ & $104.55 \pm 0.82 \mathrm{Dde}$ & $83.69 \pm 0.80 \mathrm{Fd}$ \\
Guangling & $115.07 \pm 0.97 \mathrm{Dc}$ & $130.87 \pm 0.19 \mathrm{Cc}$ & $165.15 \pm 0.42 \mathrm{Bd}$ & $173.72 \pm 0.63 \mathrm{Aa}$ & $127.77 \pm 0.89 \mathrm{Ca}$ & $94.52 \pm 0.91 \mathrm{~Eb}$ \\
Hunyuan & $126.11 \pm 0.97 \mathrm{Ca}$ & $136.26 \pm 0.79 \mathrm{Bb}$ & $163.32 \pm 0.77 \mathrm{Ad}$ & $164.96 \pm 0.70 \mathrm{Ab}$ & $126.06 \pm 0.99 \mathrm{Ca}$ & $110.88 \pm 0.89 \mathrm{Da}$ \\
Ningwu & $122.19 \pm 0.67 \mathrm{Cb}$ & $149.14 \pm 0.75 \mathrm{Ba}$ & $155.93 \pm 0.84 \mathrm{Afg}$ & $94.18 \pm 0.89 \mathrm{Dj}$ & $86.85 \pm 0.94 \mathrm{Egh}$ & $61.64 \pm 0.75 \mathrm{Fg}$ \\
Wuzhai & $98.18 \pm 0.98 \mathrm{De}$ & $111.64 \pm 0.48 \mathrm{Ce}$ & $139.55 \pm 0.46 \mathrm{Bi}$ & $144.72 \pm 0.80 \mathrm{Aef}$ & $88.60 \pm 1.01 \mathrm{Eg}$ & $47.18 \pm 0.65 \mathrm{Fi}$ \\
Tunliu & $64.80 \pm 0.75 \mathrm{Ei}$ & $107.73 \pm 0.33 \mathrm{Cf}$ & $198.59 \pm 0.44 \mathrm{Ab}$ & $160.05 \pm 0.81 \mathrm{Bc}$ & $102.39 \pm 0.60 \mathrm{De}$ & $48.22 \pm 0.91 \mathrm{Fi}$ \\
Yingxian & $90.86 \pm 1.02 \mathrm{Ef}$ & $118.12 \pm 0.85 \mathrm{Cd}$ & $129.85 \pm 0.43 \mathrm{Bj}$ & $133.61 \pm 0.53 \mathrm{Ag}$ & $112.24 \pm 0.85 \mathrm{Db}$ & $72.58 \pm 0.50 \mathrm{Fe}$ \\
Youyu & $91.82 \pm 0.89 \mathrm{Ef}$ & $128.74 \pm 0.83 \mathrm{Cc}$ & $159.00 \pm 0.48 \mathrm{Ae}$ & $146.54 \pm 0.33 \mathrm{Bde}$ & $103.94 \pm 0.08 \mathrm{De}$ & $55.96 \pm 0.80 \mathrm{Fh}$ \\
\hline
\end{tabular}

Different capital letters in the same row represent significant differences between treatments at the 0.05 level, different small letters in the same column represent significant differences between accessions at the 0.05 level.

$(P>0.05)$ difference between 'Midnight' and 'Wutai', but they were significantly $(P<0.05)$ lower in other accessions than in 'Midnight'. At 10 days of high-temperature stress, the proline contents were significantly $(P<0.05)$ lower in 'Lingqiu', and 'Guangling' than in 'Midnight', and there was no significant $(P>0.05)$ difference between 'Midnight' and 'Huozhou', but they were significantly $(P<0.05)$ higher in other accessions than in 'Midnight'. At 15 days of high-temperature stress, the proline contents were significantly $(P<0.05)$ lower in 'Tunliu' and 'Yingxian' than in 'Midnight', and there was no significant $(P>0.05)$ difference among 'Midnight', 'Huozhou', 'Xinzhou' and 'Guangling', but they were significantly $(P<0.05)$ higher in other accessions than in 'Midnight'. At 20 days of high-temperature stress, the proline contents were significantly $(P<0.05)$ higher in 'Huozhou', 'Lingshi', 'Lingqiu', 'Wuzhai' and 'Youyu' than in 'Midnight', and there was no significant $(P>0.05)$ difference between 'Midnight' and 'Ningwu', but they were significantly $(P<0.05)$ lower in other accessions than in 'Midnight'. At 25 days of high-temperature stress, the proline contents only were significantly $(P<0.05)$ higher in 'Ningwu' and 'Wuzhai' than in 'Midnight', and they were significantly $(P<0.05)$ lower in other accessions than in 'Midnight'.

\section{Changes in SOD activities of different wild Kentucky bluegrass}

The SOD activities of all the Kentucky bluegrass increased significantly $(P<0.05)$ with the enhancement of high-temperature stress, but they were also different in different accessions under same high-temperature stress (Table 5). At 0 days of high-temperature stress, the SOD activities only were significantly $(P<0.05)$ lower in 'Huozhou', 'Wutai', 'Lingshi' and 'Tunliu' than in 'Midnight', and there was no significant $(P>0.05)$ difference among 'Midnight', 'Yingxian' and 'Youyu', but they were significantly $(P<0.05)$ higher in other accessions than in 'Midnight'. At 5 days of high-temperature stress, the SOD activities only were significantly $(P<0.05)$ higher in 'Hunyuan' and 'Ningwu' than in 'Midnight', and there was no significant $(P>0.05)$ difference among 'Midnight', 'Qinshui', 'Lingqiu', 'Guangling' and 'Youyu', but they were significantly $(P<0.05)$ lower in other accessions than in 'Midnight'. At 10 days of high-temperature stress, the SOD activities only were significantly $(P<0.05)$ lower in 'Lingshi' and 'Yingxian' than in 'Midnight', and there was no significant $(P>0.05)$ difference between 'Midnight' and 'Wuzhai', but they were significantly $(P<0.05)$ higher in other accessions than in 'Midnight'. At 15 days of high-temperature stress, the SOD activities were significantly $(P<0.05)$ lower in 'Qinshui', 'Xinzhou', 'Wutai', 'Ningwu' and 'Yingxian' than in 'Midnight', and there was no significant $(P>0.05)$ difference between 'Midnight' and 'Wuzhai', but they were significantly $(P<0.05)$ higher in other accessions than in 'Midnight'. At 20 days of high-temperature stress, the SOD activities were significantly $(P<0.05)$ higher in 'Guangling', 'Hunyuan' and 'Yingxian' than in 'Midnight', and there was no significant $(P>0.05)$ difference among 'Midnight', 'Qinshui', 'Xinzhou' and 'Lingqiu', but they were significantly $(P<0.05)$ lower in other accessions than in 'Midnight'. At 25 days of high-temperature stress, the SOD activities only were significantly $(P<0.05)$ higher in 'Qinshui', 'Guangling' and 'Hunyuan' than in 'Midnight', and they were significantly $(P<0.05)$ lower in other accessions than in 'Midnight'.

\section{Changes in CAT activities of different wild Kentucky bluegrass}

The CAT activities of all the Kentucky bluegrass increased significantly $(P<0.05)$ after 0 to 15 days high-temperature stress, and then they declined significantly $(P<0.05)$ with the enhancement of high-temperature stress, but they were also different in different accessions under same high-temperature stress (Table 6). At 0 days of high-temperature stress, there was no significant $(P>0.05)$ difference between 'Midnight' and 'Hunyuan', but the CAT activities were significantly $(P<0.05)$ lower in other accessions than in 'Midnight'. At 5 days of high-temperature stress, the CAT activities were significantly $(P<0.05)$ higher in 'Qinshui', 'Wutai', 'Lingqiu', 'Hunyuan' and 'Yingxian' than in 'Midnight', and there was no significant $(P>0.05)$ difference between 'Midnight' and 'Tunliu', but they were significantly $(P<0.05)$ lower in other accessions than in 'Midnight'. At 10 days of high-temperature stress, the CAT activities only were significantly $(P<0.05)$ higher in 'Qinshui', 'Wutai', 'Lingqiu', 'Hunyuan' and 'Yingxian' than in 'Midnight', and they were significantly $(P<0.05)$ lower in other accessions than in 'Midnight'. At 15 days of 
TABLE 6. Effects of high-temperature stress on CAT activities in leaves of different wild Kentucky bluegrass $\left(\mu \mathrm{mol} \mathrm{min}^{-1} \mathrm{mg}^{-1}\right.$ protein).

\begin{tabular}{llccccc}
\hline \multirow{2}{*}{ Accessions } & \multicolumn{5}{c}{ Duration of high-temperature stress (days) } \\
\cline { 2 - 7 } & \multicolumn{1}{c}{0} & 5 & 10 & 15 & 20 & 25 \\
\hline Midnight & $9.82 \pm 0.057 \mathrm{Ea}$ & $11.00 \pm 0.047 \mathrm{De}$ & $13.12 \pm 0.022 \mathrm{Cf}$ & $14.65 \pm 0.029 \mathrm{Ad}$ & $13.87 \pm 0.032 \mathrm{Bc}$ & $7.65 \pm 0.045 \mathrm{Fa}$ \\
Qinshui & $8.48 \pm 0.061 \mathrm{Ecd}$ & $12.30 \pm 0.044 \mathrm{Db}$ & $14.88 \pm 0.009 \mathrm{Cb}$ & $16.40 \pm 0.012 \mathrm{Ab}$ & $15.74 \pm 0.069 \mathrm{Ba}$ & $7.06 \pm 0.063 \mathrm{Fc}$ \\
Huozhou & $9.12 \pm 0.034 \mathrm{~Eb}$ & $9.16 \pm 0.016 \mathrm{Dj}$ & $10.63 \pm 0.023 \mathrm{Bm}$ & $11.55 \pm 0.011 \mathrm{Al}$ & $9.68 \pm 0.021 \mathrm{Ck}$ & $7.31 \pm 0.025 \mathrm{Fb}$ \\
Xinzhou & $7.01 \pm 0.059 \mathrm{Df}$ & $10.41 \pm 0.026 \mathrm{Cg}$ & $11.82 \pm 0.015 \mathrm{Bj}$ & $12.74 \pm 0.020 \mathrm{Aj}$ & $11.69 \pm 0.018 \mathrm{Bh}$ & $4.44 \pm 0.062 \mathrm{Eh}$ \\
Wutai & $8.42 \pm 0.060 \mathrm{Ed}$ & $11.64 \pm 0.045 \mathrm{Dc}$ & $13.70 \pm 0.018 \mathrm{Bd}$ & $14.55 \pm 0.029 \mathrm{Ae}$ & $11.87 \pm 0.019 \mathrm{Cg}$ & $6.00 \pm 0.064 \mathrm{Fd}$ \\
Lingshi & $7.70 \pm 0.058 \mathrm{Ee}$ & $9.63 \pm 0.047 \mathrm{Di}$ & $11.22 \pm 0.020 \mathrm{Bl}$ & $12.11 \pm 0.040 \mathrm{Ak}$ & $10.85 \pm 0.012 \mathrm{Cj}$ & $5.17 \pm 0.058 \mathrm{Ff}$ \\
Lingqiu & $7.71 \pm 0.055 \mathrm{Ee}$ & $11.35 \pm 0.035 \mathrm{Dd}$ & $13.24 \pm 0.026 \mathrm{Be}$ & $14.15 \pm 0.038 \mathrm{Af}$ & $12.28 \pm 0.019 \mathrm{Cf}$ & $4.83 \pm 0.055 \mathrm{Fg}$ \\
Guangling & $8.36 \pm 0.029 \mathrm{Ed}$ & $9.88 \pm 0.058 \mathrm{Dh}$ & $12.25 \pm 0.028 \mathrm{Ch}$ & $14.21 \pm 0.018 \mathrm{Af}$ & $13.53 \pm 0.043 \mathrm{Bd}$ & $7.07 \pm 0.046 \mathrm{Fc}$ \\
Hunyuan & $9.82 \pm 0.057 \mathrm{Ea}$ & $12.61 \pm 059 \mathrm{Da}$ & $15.16 \pm 0.042 \mathrm{Ca}$ & $16.69 \pm 0.015 \mathrm{Aa}$ & $15.42 \pm 0.064 \mathrm{Bb}$ & $7.77 \pm 0.061 \mathrm{Fa}$ \\
Ningwu & $7.01 \pm 0.059 \mathrm{Ef}$ & $10.42 \pm 0.021 \mathrm{Dg}$ & $11.96 \pm 0.009 \mathrm{Bi}$ & $12.83 \pm 0.013 \mathrm{Aj}$ & $11.20 \pm 0.015 \mathrm{Ci}$ & $4.47 \pm 0.050 \mathrm{Fh}$ \\
Wuzhai & $6.29 \pm 0.024 \mathrm{Eg}$ & $9.96 \pm 0.043 \mathrm{Dh}$ & $11.69 \pm 0.013 \mathrm{Bk}$ & $13.22 \pm 0.009 \mathrm{Ah}$ & $11.25 \pm 0.026 \mathrm{Ci}$ & $4.92 \pm 0.046 \mathrm{Fg}$ \\
Tunliu & $7.69 \pm 0.046 \mathrm{Ee}$ & $10.91 \pm 0.004 \mathrm{De}$ & $12.26 \pm 0.010 \mathrm{Bh}$ & $13.05 \pm 0.034 \mathrm{Ai}$ & $11.74 \pm 0.032 \mathrm{Cgh}$ & $5.59 \pm 0.074 \mathrm{Fe}$ \\
Yingxian & $8.65 \pm 0.025 \mathrm{Ebc}$ & $12.69 \pm 0.035 \mathrm{Da}$ & $14.28 \pm 0.009 \mathrm{Bc}$ & $15.02 \pm 0.023 \mathrm{Ac}$ & $12.94 \pm 0.012 \mathrm{Ce}$ & $6.09 \pm 0.030 \mathrm{Fd}$ \\
Youyu & $8.44 \pm 0.021 \mathrm{Ed}$ & $10.61 \pm 0.058 \mathrm{Df}$ & $12.54 \pm 0.052 \mathrm{Cg}$ & $13.46 \pm 0.029 \mathrm{Ag}$ & $12.82 \pm 0.020 \mathrm{Be}$ & $5.49 \pm 0.041 \mathrm{Fe}$ \\
\hline
\end{tabular}

Different capital letters in the same row represent significant differences between treatments at the 0.05 level, different small letters in the same column represent significant differences between accessions at the 0.05 level.

high-temperature stress, the CAT activities in 'Wutai' and 'Lingqiu' also changed to lower than in 'Midnight', and they still remained unchanged in other accessions comparing to 'Midnight'. At 20 days of high-temperature stress, the CAT activities only were significantly $(P<0.05)$ higher in 'Qinshui' and 'Hunyuan' than in 'Midnight', and they were significantly $(P<0.05)$ lower in other accessions than in 'Midnight'. At 25 days of high-temperature stress, except there was no significant $(P>0.05)$ difference between 'Midnight' and 'Hunyuan', the CAT activities were all significantly $(P<0.05)$ lower in other accessions than in 'Midnight'.

\section{Changes in MDA contents of different wild Kentucky bluegrass}

The MDA contents of all the Kentucky bluegrass increased significantly $(P<0.05)$ with the enhancement of high-temperature stress, but they were also different in different accessions under same high-temperature stress (Table 7). At 0 days of high-temperature stress, the MDA contents were significantly $(P<0.05)$ higher in 'Huozhou', 'Guangling', 'Hunyuan', 'Ningwu' and 'Yingxian' than in 'Midnight', and there was no significant $(P>0.05)$ difference among 'Midnight', 'Xinzhou' and 'Wutai', but they were significantly $(P<0.05)$ lower in other accessions than in 'Midnight'. At 5 days of high-temperature stress, the MDA contents were significantly $(P<0.05)$ higher in 'Huozhou', 'Xinzhou', 'Wutai' and 'Yingxian' than in 'Midnight', and there was no significant $(P>0.05)$ difference among 'Midnight', 'Guangling,', 'Hunyuan' and 'Wuzhai', but they were significantly $(P<0.05)$ lower in other accessions than in 'Midnight'. At 10 days of high-temperature stress, except there was no significant $(P>0.05)$ difference between 'Midnight' and 'Hunyuan', the MDA con-

TABLE 7. Effects of high-temperature stress on MDA contents in leaves of different wild Kentucky bluegrass ( $\mu$ mol g-1 FW).

\begin{tabular}{lcccccc}
\hline \multirow{2}{*}{ Accessions } & \multicolumn{5}{c}{ Duration of high-temperature stress (days) } \\
\cline { 2 - 6 } & 0 & 5 & 10 & 15 & 20 & 25 \\
\hline Midnight & $13.12 \pm 0.022 \mathrm{Cf}$ & $4.01 \pm 0.032 \mathrm{Ed}$ & $4.31 \pm 0.029 \mathrm{Dg}$ & $8.01 \pm 0.055 \mathrm{Cf}$ & $10.36 \pm 0.022 \mathrm{Bf}$ & $11.52 \pm 0.020 \mathrm{Ag}$ \\
Qinshui & $14.88 \pm 0.009 \mathrm{Cb}$ & $3.82 \pm 0.035 \mathrm{Eef}$ & $5.35 \pm 0.062 \mathrm{De}$ & $6.80 \pm 0.050 \mathrm{Ci}$ & $7.55 \pm 0.049 \mathrm{Bk}$ & $9.23 \pm 0.040 \mathrm{Ak}$ \\
Huozhou & $10.63 \pm 0.023 \mathrm{Bm}$ & $4.78 \pm 0.020 \mathrm{Ea}$ & $6.30 \pm 0.072 \mathrm{Db}$ & $9.49 \pm 0.012 \mathrm{Cd}$ & $11.80 \pm 0.032 \mathrm{Bb}$ & $12.73 \pm 0.044 \mathrm{Ad}$ \\
Xinzhou & $3.33 \pm 0.046 \mathrm{Ed}$ & $4.40 \pm 0.046 \mathrm{Db}$ & $5.07 \pm 0.024 \mathrm{Cf}$ & $10.08 \pm 0.043 \mathrm{Bb}$ & $10.21 \pm 0.067 \mathrm{Bg}$ & $11.08 \pm 0.047 \mathrm{Ah}$ \\
Wutai & $3.25 \pm 0.027 \mathrm{Fde}$ & $4.55 \pm 0.059 \mathrm{~Eb}$ & $6.47 \pm 0.044 \mathrm{Db}$ & $9.94 \pm 0.058 \mathrm{Cbc}$ & $11.23 \pm 0.020 \mathrm{Bd}$ & $11.98 \pm 0.049 \mathrm{Af}$ \\
Lingshi & $2.87 \pm 0.036 \mathrm{Fh}$ & $3.78 \pm 0.048 \mathrm{Eef}$ & $5.25 \pm 0.023 \mathrm{Def}$ & $7.40 \pm 0.036 \mathrm{Ch}$ & $9.64 \pm 0.043 \mathrm{Bh}$ & $10.49 \pm 0.029 \mathrm{Ai}$ \\
Lingqiu & $2.94 \pm 0.044 \mathrm{Fh}$ & $3.73 \pm 0.046 \mathrm{Ef}$ & $6.94 \pm 0.045 \mathrm{Da}$ & $10.21 \pm 0.027 \mathrm{Ca}$ & $11.65 \pm 0.044 \mathrm{Bc}$ & $12.05 \pm 0.044 \mathrm{Af}$ \\
Guangling & $3.65 \pm 0.041 \mathrm{Fb}$ & $4.07 \pm 0.023 \mathrm{Ecd}$ & $6.30 \pm 0.038 \mathrm{Db}$ & $9.93 \pm 0.060 \mathrm{Cbc}$ & $10.61 \pm 0.045 \mathrm{Be}$ & $11.38 \pm 0.019 \mathrm{Ag}$ \\
Hunyuan & $3.49 \pm 0.013 \mathrm{Fc}$ & $3.94 \pm 0.032 \mathrm{Ede}$ & $4.42 \pm 0.071 \mathrm{Dg}$ & $7.74 \pm 0.086 \mathrm{Cg}$ & $9.46 \pm 0.028 \mathrm{Bi}$ & $10.21 \pm 0.026 \mathrm{Aj}$ \\
Ningwu & $3.66 \pm 0.024 \mathrm{Fb}$ & $4.20 \pm 0.038 \mathrm{Ec}$ & $5.62 \pm 0.072 \mathrm{Dcd}$ & $8.78 \pm 0.053 \mathrm{Ce}$ & $9.27 \pm 0.035 \mathrm{Bj}$ & $12.28 \pm 0.051 \mathrm{Ae}$ \\
Wuzhai & $2.89 \pm 0.009 \mathrm{Fh}$ & $3.94 \pm 0.041 \mathrm{Ede}$ & $5.47 \pm 0.070 \mathrm{Dde}$ & $6.90 \pm 0.021 \mathrm{Ci}$ & $11.72 \pm 0.041 \mathrm{Bbc}$ & $14.48 \pm 0.035 \mathrm{Aa}$ \\
Tunliu & $3.15 \pm 0.022 \mathrm{Fef}$ & $4.19 \pm 0.033 \mathrm{Ec}$ & $6.95 \pm 0.035 \mathrm{Da}$ & $7.78 \pm 0.029 \mathrm{Cg}$ & $10.14 \pm 0.016 \mathrm{Bg}$ & $13.14 \pm 0.046 \mathrm{Ac}$ \\
Yingxian & $3.89 \pm 0.029 \mathrm{Fa}$ & $4.54 \pm 0.035 \mathrm{~Eb}$ & $6.25 \pm 0.044 \mathrm{Db}$ & $8.86 \pm 0.066 \mathrm{Ce}$ & $12.74 \pm 0.021 \mathrm{Ba}$ & $13.96 \pm 0.038 \mathrm{Ab}$ \\
Youyu & $2.97 \pm 0.024 \mathrm{Fgh}$ & $3.66 \pm 0.036 \mathrm{Ef}$ & $5.74 \pm 0.055 \mathrm{Dc}$ & $9.78 \pm 0.094 \mathrm{Cc}$ & $10.45 \pm 0.028 \mathrm{Bf}$ & $12.26 \pm 0.042 \mathrm{Ae}$ \\
\hline
\end{tabular}

Different capital letters in the same row represent significant differences between treatments at the 0.05 level, different small letters in the same column represent significant differences between accessions at the 0.05 level. 
tents were significantly $(P<0.05)$ higher in other accessions than in 'Midnight'. At 15 days of high-temperature stress, the MDA contents were significantly $(P<0.05)$ lower in 'Qinshui', 'Lingshi', 'Hunyuan', 'Wuzhai' and 'Tunliu' than in 'Midnight', and they were significantly $(P<0.05)$ higher in other populations than in 'Midnight'. At 20 days of high-temperature stress, the proline contents were significantly $(P<0.05)$ higher in 'Huozhou', 'Wutai', 'Lingqiu', 'Guangling', 'Wuzhai' and 'Yingxian' than in 'Midnight', and there was no significant $(P>0.05)$ difference between 'Midnight' and 'Youyu', but they were significantly $(P<0.05)$ lower in other accessions than in 'Midnight'. At 25 days of high-temperature stress, the MDA contents only were significantly $(P<0.05)$ lower in 'Qinshui', 'Xinzhou', 'Lingshi' and 'Hunyuan' than in 'Midnight', and there was no significant $(P>0.05)$ difference between 'Midnight' and 'Guangling', they were significantly $(P<0.05)$ higher in other accessions than in 'Midnight'.

\section{Discussion}

Disequilibrium of cellular homeostasis is the major cause for oxidative damage to membrane, lipids, proteins and nucleic acids in plants by environmental stresses (Srivalli et al., 2003). Lipid peroxidation, symbolizing with the production of MDA, is an important manifestation of environmental stresses in plants (Gupta et al., 2013). Thus, the MDA contents often augmented prominently in plants under high-temperature stress (Gao et al., 2010). Uniformly, the obvious increase of lipid peroxidation had also been observed in Kentucky bluegrass during high-temperature stress (Wang and Huang, 2004; He et al., 2005). In our study, the MDA contents increased significantly $(P<0.05)$ in all the accessions of Kentucky bluegrass along with the enhancement of high-temperature stress (Table 7), manifesting that lipid peroxidation was enhanced markedly in all the accessions of Kentucky bluegrass under high-temperature stress.

Osmotic adjustment is an important pathway to resist damage from environmental stress in plants (Sangu et al., 2015). Particularly, the accumulation of osmoprotectants, e.g., proline, sugars, proteins, were often regarded as a primary strategy for the protection and survival of plants under environmental stress (Chen et al., 2007). Under high-temperature stress, a remarkable enhancement of the soluble sugar and proline contents was observed in many plants for their osmotic adjustment (Sassi-Aydi et al., 2014; Harsh et al., 2016). In this study, the soluble sugar and proline contents also increased significantly $(P<0.05)$ in all Kentucky bluegrass along with the enhancement of high-temperature stress (Tables 2 and 4), indicating that osmotic adjustment capacity was augmented in Kentucky bluegrass to resist their injury from high-temperature stress. Similar results had been observed in Kentucky bluegrass under high-temperature stress (Du et al., 2011). Nonetheless, the soluble protein contents of all the Kentucky bluegrass decreased significantly $(P<0.05)$ during 5 to 10 days of high-temperature stress in our study (Table 3); this might be due to the inhibition of protein synthesis and the denaturation of some proteins at the initial stage of high-temperature stress (Levitt, 1980). Similarly, a remarkable decline of total soluble protein contents had been reported in leaves of mulberry (Morus alba; Chaitanya et al., 2001) and strawberry (Fragaria $\times$ ananassa; Gulen and Eris, 2004) under high-temperature stress. Conversely, the soluble protein contents of all the Kentucky bluegrass increased significantly $(P<0.05)$ during 15 to 25 days of high-temperature stress; this might be due to the release of membrane proteins by enhancement of lipid peroxidation and proteins degradation at severe high-temperature stress (Gulen and Eris, 2004). In our study, The MDA contents of all the Kentucky bluegrass increased sharply during 15 to 25 days of high-temperature stress (Table 7), and the levels of SOD and CAT activities also declined significantly $(P<0.05)$ during this period of high-temperature stress (Tables 5 and 6). Thereby, the increase in soluble protein contents of Kentucky bluegrass might not be related to the enhancement of their osmotic adjustment ability, but it might be manifested their severe damage caused by high-temperature stress.

High levels of antioxidant enzymes had been observed in many plants during response to high-temperature stress, and they might have a specific role in the acquisition of tolerance to high-temperature stress (Tutar et al., 2017). The high levels of SOD and CAT activities played an important role in Kentucky bluegrass for response to high-temperature stress (Wang and Huang, 2004; He et al., 2005; He and Huang, 2010). In this study, the SOD activities also increased significantly $(P<0.05)$ in most accessions of Kentucky bluegrass during 0 to 15 days of high-temperature stress (Table $5)$, and the CAT activities increased significantly $(P<0.05)$ in all the accessions of Kentucky bluegrass during 0 to 15 days of high-temperature stress (Table 6). Simultaneously, The MDA contents increased significantly, but remained at a relatively low level (Table 7). Analogously, Harsh et al. (2016) had also observed that a prominent increase of SOD and CAT activities in most genotypes of moth bean (Vigna aconitifolia) accompanying with a remarkable over-accumulation of total sugar and proline under short-term high-temperature stress. These results showed that the high level of SOD and CAT activities delayed the explosion of lipid peroxidation in Kentucky bluegrass under mild and moderate high-temperature stress. However, the SOD and CAT activities declined significantly $(P<0.05)$ in all the accessions of Kentucky bluegrass during 15 to 25 days of high-temperature stress (Tables 5 and 6), and the MDA contents also produced rapidly in all the accessions of Kentucky bluegrass (Table 7). Similarly, an explosion of lipid peroxidation was also observed in many plants under high-temperature stress (Wang and Huang, 2004; Gao et al., 2007). Consequently, these results indicated that the action of SOD and CAT played an important role for Kentucky bluegrass in resisting high-temperature stress.

The defense capability was often diverse in different genotypes of plants to resist high-temperature stress, and their activation levels were also disparate in different genotypes of plants with the enhancement of high-temperature stress (Almeselmani et al., 2006). Under high-temperature stress, the variation of antioxidant capacity and lipid peroxidation was also different in different Kentucky bluegrass, which indicated that their responses to high-temperature stress were different (Wang and Huang, 2004; He, 2010; Du et al., 2011). In this study, the MDA contents of Kentucky bluegrass remained at a relatively low level in 'Qinshui', 'Lingshi' and 'Hunyuan' under high-temperature stress, but remained at a relatively high level in 'Huozhou', 'Wutai' and 'Yingxian', which symbolized that lipid peroxidation of Kentucky bluegrass in 'Qinshui', 'Lingshi' and 'Hunyuan' was more temperate than in 'Huozhou', 'Wutai' and 'Yingxian' under high-temperature stress. Under oxidative stress, $\mathrm{O}_{2}{ }^{-}$generated by the electron transport chain, the first to cause lipid peroxidation, often was dismutated to $\mathrm{H}_{2} \mathrm{O}_{2}$ by SOD in the matrix of plants (Matamoros et al., 2013). In this study, the SOD activities of Kentucky bluegrass still held at a relatively high level in 'Qinshui', 'Guangling' and 'Hunyuan' under high-temperature stress, but they held at a relatively low level in 'Wutai' and 
'Lingshi', which manifested that more $\mathrm{O}_{2}^{-}$was dismutated to $\mathrm{H}_{2} \mathrm{O}_{2}$ by SOD in 'Qinshui', 'Guangling' and 'Hunyuan' than in 'Wutai' and 'Lingshi'. However, the CAT activities of Kentucky bluegrass maintained at a relatively high level in 'Qinshui', 'Hunyuan' and 'Yingxian' under high-temperature stress, but they maintained at a relatively low level in 'Huozhou', 'Xinzhou', 'Lingshi', 'Guangling', 'Ningwu', 'Wuzhai', 'Tunliu' and 'YouYu', showing that more $\mathrm{H}_{2} \mathrm{O}_{2}$ was decomposed into $\mathrm{H}_{2} \mathrm{O}$ by CAT in 'Qinshui', 'Hunyuan' and 'Yingxian' than in 'Huozhou', 'Xinzhou', 'Lingshi', 'Guangling', 'Ningwu', 'Wuzhai', 'Tunliu' and 'YouYu'. Similarly, the changes in SOD and CAT activities were also different in 37 genotypes of moth bean under short-term high-temperature stress (Harsh et al., 2016). In this study, the soluble protein contents of Kentucky bluegrass retained at a relatively high level in 'Qinshui', 'Hunyuan' and 'Yingxian' under high-temperature stress, but they retained at a relatively low level in 'Huozhou', 'Xinzhou', 'Wutai', 'Ningwu' and 'Tunliu'; this indicated that more protection might be provided by the enhancement of soluble protein contents in 'Qinshui', 'Hunyuan' and 'Yingxian' than in 'Huozhou', 'Xinzhou', 'Wutai', 'Ningwu' and 'Tunliu' under high-temperature stress. Moreover, the proline contents of Kentucky bluegrass sustained at a relatively high level in 'Qinshui', 'Hunyuan' and 'Wuzhai' under high-temperature stress, but they sustained at a relatively low level in 'Xinzhou', 'Guangling' and 'Yingxian'; this result also showed that there might be a higher capacity for osmotic regulation in 'Qinshui', 'Hunyuan' and 'Wuzhai' than in 'Xinzhou', 'Guangling' and 'Yingxian' under high-temperature stress. Likewise, the soluble sugar contents of Kentucky bluegrass kept at a relatively high level in 'Huozhou', 'Wutai' and 'Tunliu' under high-temperature stress, but they kept at a relatively low level in 'Xinzhou', 'Lingshi', 'Yingxian' and 'Youyu'; this result suggested that the ability of osmotic regulation might be enhanced in 'Huozhou', 'Wutai' and 'Tunliu' than in 'Xinzhou', 'Lingshi', 'Yingxian' and 'Youyu' under high-temperature stress. Consequently, there were different osmotic and antioxidative protection responses to high-temperature stress in different accessions of Kentucky bluegrass. Whereas, the MDA contents of Kentucky bluegrass kept at a relatively low level in 'Qinshui' and 'Hunyuan' under high-temperature stress, both their proline and soluble protein contents and activities of SOD and CAT all maintained at a high level, there results showed that they had a high adaptability to high-temperature stress.

\section{Conclusions}

In summary, the contents of MDA, proline and soluble sugar increased in all accessions of Kentucky bluegrass with the enhancement of high-temperature stress, the soluble protein contents decreased in all accessions of Kentucky bluegrass under 0 to 10 days of high-temperature stress, but they increased after 15 days of high-temperature stress. On the contrary, the activities of SOD and CAT increased in all accessions of Kentucky bluegrass under 10 or 15 days of high-temperature stress, and then declined with the continuous enhancement of high-temperature stress. The osmotic and antioxidant responses to high-temperature stress were different in different accessions of Kentucky bluegrass, but the decrease of SOD and CAT activities in 'Qinshui' and 'Hunyuan' were relatively slow, and their MDA contents also increased slowly. Therefore, 'Qinshui' and 'Hunyuan' accessions of Kentucky bluegrass had a high resistance to high-temperature stress, which could be used to develop new varieties which were resistant to high-temperature stress.

\section{Acknowledgments}

This work was financially supported by the Key Research Development Program of Shanxi Province (No. 201603 D221017-2).

\section{References}

Almeselmani, M., Deshmukh, P.S., Sairam, R.K., Kushwaha, S.R., and Singh, T.P. (2006). Protective role of antioxidant enzymes under high temperature stress. Plant Sci. 171, 382-388. https://doi. org/10.1016/j.plantsci.2006.04.009.

Bailly, C., Benamar, A., Corbineau, F., and Côme, D. (1996). Changes in malondialdehyde content and superoxide dismutase, catalase and glutathione reductase activities in sunflower seeds as related to deterioration during accelerated aging. Physiol. Plant. 97, 104-110. https://doi.org/10.1111/j.1399-3054.1996.tb00485.x.

Bates, L.S., Waldren, R.P., and Teare, I.D. (1973). Rapid determination of free proline for water-stress studies. Plant Soil 39, 205-207. https://doi.org/10.1007/BF00018060.

Bonos, S., and Murphy, J. (1999). Growth responses and performance of Kentucky bluegrass under summer stress. Crop Sci. 39, 770-774. https://doi.org/10.2135/cropsci1999.0011183X003900030026x.

Bradford, M.M. (1976). A rapid and sensitive method for the quantitation of microgram quantities of protein utilizing the principle of protein-dye binding. Anal. Biochem. 72, 248-254. https://doi.org/10.1016/0003-2697(76)90527-3.

Chaitanya, K.V., Sundar, D., and Reddy, A.R. (2001). Mulberry leaf metabolism under high temperature stress, Biol. Plant. 44, 379-384. https://doi.org/10.1023/A:1012446811036.

Chance, B., and Maehly, A.C. (1955). Assay of catalase and peroxidase. Method. Enzymol. 2, 764-775. https://doi.org/10.1016/S00766879(55)02300-8.

Chen, Z., Cuin, T.A., Zhou, M., Twomey, A., Naidu, B.P., and Shabala, S. (2007). Compatible solute accumulation and stress-mitigating effects in barley genotypes contrasting in their salt tolerance. J. Exp. Bot. 58, 4245-4255. https://doi.org/10.1093/jxb/erm284.

Du, H., Wang, Z., Yu, W., Liu, Y., and Huang, B. (2011). Differential metabolic responses of perennial grass Cynodon transvaalensis $\times$ Cynodon dactylon (C4) and Poa pratensis (C3) to heat stress. Physiol. Plant. 141, 251-264. https://doi:10.1111/j.13993054.2010.01432.x.

Dubois, M., Gilles, K., Hamilton, J.K., Robers, P.A., and Smith, F. (1956). A colorimetric method for the determination of sugar and related substances. Anal. Chem. 28, 350-356. https://doi.org/10.1021/ ac60111a017.

Ergo, V.V., Lascano, R., Vega, C.R.C., Parola, R., and Carrera, C.S. (2018). Heat and water stressed field-grown soybean: A multivariate study on the relationship between physiological-biochemical traits and yield. Environ. Exp. Bot. 148, 1-11. https://doi.org/10.1016/j. envexpbot.2017.12.023.

Fang, C., Dou, L., Liu, Y., Yu, J., and Tu, J. (2018). Heat stress-responsive transcriptome analysis in heat susceptible and tolerant rice by highthroughput sequencing. Ecol. Genetics and Genomics 6, 33-40. https://doi.org/10.1016/j.egg.2017.12.001.

Gao, Y., Guo, Y.K., Lin, S.H., Fang, Y.Y., and Bai, J.G. (2010). Hydrogen peroxide pretreatment alters the activity of antioxidant enzymes and protects chloroplast ultrastructure in heat-stressed cucumber leaves. Sci. Hortic. 126, 20-26. https://doi.org/10.1016/j. scienta.2010.06.006.

Gourdji, S.M., Sibley, A.M., and Lobell, D.B. (2013). Global crop exposure to critical high temperatures in the reproductive period: Historical trends and future projections. Environ. Res. Lett. 8, 24-41. https://doi.org/10.1088/1748-9326/8/2/024041. 
Gulen, H., and Eris, A. (2004). Effect of heat stress on peroxidase activity and total protein content in strawberry plants. Plant Sci. 166, 739-744. https://doi.org/10.1016/j.plantsci.2003.11.014.

Gupta, N.K., Agarwal, S., Agarwal, V.P., Nathawat, N.S., Gupta, S., and Singh, G. (2013). Effect of short-term heat stress on growth, physiology and antioxidative defence system in wheat seedlings. Acta Physiol. Plant. 35, 1837-1842. https://doi.org/10.1007/ s11738-013-1221-1.

Harsh, A., Sharma, Y.K., Joshi, U., Rampuria, S., Singh, G., Kumar, S., and Sharma, R. (2016). Effect of short-term heat stress on total sugars, proline and some antioxidant enzymes in moth bean (Vigna aconitifolia). Ann. Agric. Sci. 61, 57-64. https://doi.org/10.1016/j. aoas.2016.02.001.

He, Y., and Huang, B. (2010). Differential responses to heat stress in activities and isozymes of four antioxidant enzymes for two cultivars of Kentucky bluegrass contrasting in heat tolerance. J. Am. Soc. Hortic. Sci. 135, 116-124. https://doi.org/10.21273/JASHS.135.2.116.

He, Y., Liu, Y., Cao, W., Huai, M., Xu, B., and Huang, B. (2005). Effects of salicylic acid on heat tolerance associated with antioxidant metabolism in Kentucky bluegrass. Crop Sci. 45, 988-995. https:// doi:10.2135/cropsci2003.0678.

Kibinza, S., Vinel, D., Côme, D., Bailly, C., and Corbineau, F. (2006). Sunflower seed deterioration as related to moisture content during ageing, energy metabolism and active oxygen species scavenging. Physiol. Plant. 128, 496-506. http:// doi.org/10.1111/j.13993054.2006.00771.x.

Kotak, S., Larkindale, J., Lee, U., von Koskull-Döring, P., Vierling, E., and Scharf, K. (2007). Complexity of the heat stress response in plants. Curr. Opin. Plant Biol. 10, 310-316. https://doi.org/10.1016/j. pbi.2007.04.011.

Kraus, T.E., McKersie, B.D., and Fletcher, R.A. (1995). Paclobutrazole induced tolerance of wheat leaves to paraquat may involve antioxidant enzyme activity. J. Plant Physiol. 145, 570-576. https:// doi.org/10.1016/S0176-1617(11)81790-6.

Levitt, J. (1980). Responses of Plants to Environmental Stresses, Vol. I (New York: Academic Press), p. 347-470.

Matamoros, M.A., Fernández-García, N., Wienkoop, S., Loscos, J., Saiz, A., and Becana, M. (2013). Mitochondria are an early target of oxidative modifications in senescing legume nodules. New Phytol. 197, 873-885. https://doi.org/10.1111/nph.12049.

Mittler, R., Vanderauwera, S., Suzuki, N., Miller, G., Tognetti, V.B., Vandepoele, K., Gollery, M., Shulaev, V., and Van Breusegem, F. (2011). ROS signaling: the new wave? Trends Plant Sci. 16, 300-309. https:// doi.org/10.1016/j.tplants.2011.03.007.

Rao, K.V.M., and Sresty, T.V.S. (2000). Antioxidant parameters in the seedlings of pigeon pea (Cajanus cajan (L.) Millspaugh) in response to Zn and Ni stresses. Plant Sci. 157, 113-128. https://doi. org/10.1016/S0168-9452(00)00273-9.

Rezaei, E.E., Webber, H., Gaiser, T., Naab, J., and Ewert, F. (2015). Heat stress in cereals: Mechanisms and modelling. Eur. J. Agron. 64, 98113. https://doi.org/10.1016/j.eja.2014.10.003.

Sangu, E., Tibazarwa, F.I., Nyomora, A., and Symonds, R.C. (2015). Expression of genes for the biosynthesis of compatible solutes during pollen development under heat stress in tomato (Solanum lycopersicum). J. Plant Physiol. 178, 10-16. https://doi. org/10.1016/j.jplph.2015.02.002.

Sassi-Aydi, S., Aydi, S., and Abdelly, C. (2014). Inorganic nitrogen nutrition enhances osmotic stress tolerance in Phaseolus vulgaris: Lessons from a drought-sensitive cultivar. HortScience 49, 550-555. https://doi.org/10.21273/HORTSCI.49.5.550.
Srivalli, B., Vishanathan, C., and Renu, K.C. (2003). Antioxidant defense in response to abiotic stresses in plants. J. Plant Biol. 30, 121-139.

Tutar, O., Marín-Guirao, L., Ruiz, J.M., and Procaccini, G. (2017). Antioxidant response to heat stress in seagrasses. A gene expression study. Mar. Environ. Res. 132, 94-102. https://doi.org/10.1016/j. marenvres.2017.10.011.

Wang, Z., and Huang, B. (2004). Physiological recovery of Kentucky bluegrass from simultaneous drought and heat stress. Crop Sci. 44, 1729-1736. https://doi.org/10.2135/cropsci2004.1729.

Webber, H., Martre, P., Asseng, S., Kimball, B., White, J., Ottman, M., Wall, G.W., De Sanctis, G., Doltra, J., Grant, R., Kassie, B., Maiorano, A., Olesen, J.E., Ripoche, D., Rezaei, E.E., Semenov, M.A., Stratonovitch, P., and Ewert, F. (2017). Canopy temperature for simulation of heat stress in irrigated wheat in a semi-arid environment: A multi-model comparison. Field Crop Res. 202, 21-35. https://doi.org/10.1016/j. fcr.2015.10.009.

Wu, S.W., Hu, C.X., Tan, Q., Nie, Z.J., and Sun, X.C. (2014). Effects of molybdenum on water utilization, antioxidative defense system and osmotic adjustment ability in winter wheat (Triticum aestivum) under drought stress. Plant Physiol. Biochem. 83, 365-374. https:// doi.org/10.1016/j.plaphy.2014.08.022.

Zou, M., Yuan, L., Zhu, S., Liu, S., Ge, J., and Wang, C. (2016). Response of osmotic adjustment and ascorbate-glutathione cycle to heat stress in a heat-sensitive and a heat-tolerant genotype of wucai (Brassica campestris L.). Sci. Hortic. 211, 87-94. https://doi.org/10.1016/j. scienta.2016.08.011.

Received: Sep. 29, 2018

Accepted: Apr. 6, 2020

Address of authors:

Fangshan Xia, Zhaosheng Han, Huisen Zhu*, Kuanhu Dong and Lixia Du

College of Grassland Science, Shanxi Agricultural University,

Taigu, Shanxi 030801, China

* Corresponding author; E-mail: zhuhuisen@126.com

Tel.: 86+0354-6287563; Fax: 86+0354-6287563 